\title{
Kompetenz, Selbstwirksamkeitserwartung und die Rolle von Vorbildern in der Ordnungsethik ${ }^{*}$
}

\author{
MichaEl VON GRUNDHERR ${ }^{* *}$
}

Nach der zentralen Forderung der Ordnungsethik müssen moralische Normen durch formale und informelle Institutionen, die moralisches Handeln anreizkompatibel machen, implementiert werden. Als eine minimale (normative) Bedingung müssen diese Institutionen in der Lage sein, den Homo oeconomicus zu motivieren. Aber auch wenn eine Institution diese Bedingung erfüllt, wird sie nur diejenigen realen Personen zum moralischen Handeln motivieren, die über die relevanten Kompetenzen und Selbstwirksamkeitsüberzeugungen verfügen. An dieser Stelle kann die angewandte Ordnungsethik auf Befunde aus der Psychologie und der experimentellen Ökonomik zurückgreifen. Man kann zeigen, dass Vorbilder Selbstwirksamkeitsüberzeugungen stützen können und somit aus Sicht der Ordnungsethik wichtiger sind, als deren Vertreter traditioneller Weise annehmen.

Schlagwörter: Ordnungsethik, Selbstwirksamkeit, Moralpsychologie, Institutionen, Whistleblowing, Vorbilder

\section{The Importance of Moral Competence, Self-efficacy and Role Models for Order Ethics}

According to the order ethics approach to business ethics, moral rules must be implemented by formal and informal institutions, which provide incentives for acting morally. As a minimal (normative) condition, these institutions must be able to motivate the homo economicus. But even if an institution passes this test, it will only motivate actual people (i.e. the homo psychologicus) to follow moral rules, if they have the relevant competences and self-efficacy beliefs. At this point applied order ethics can draw on findings of psychology and experimental economics. It turns out, that role models can support self-efficacy beliefs and are thus more important for order ethics than has traditionally been assumed.

Keywords: Order Ethics, Self-Efficacy, Moral Psychology, Institutions, Whistleblowing, Role Models

\footnotetext{
* Beitrag eingereicht am 30.11.2013, nach doppelt verdecktem Gutachterverfahren überarbeitete Fassung angenommen am 14.04.2014.

** Dr. Michael von Grundherr, Ludwig-Maximilians-Universität München, Forschungsstelle Neurophilosophie und Ethik der Neurowissenschaften, Geschwister-Scholl-Platz 1, 80539 München, Tel.:+49-(0)892180 9889, E-Mail: mvg@lrz.uni-muenchen.de, Forschungsschwerpunkte: Wirtschaftsethik, Moralpsychologie, Neurophilosophie, Metaethik.
} 


\section{Einleitung}

Mitarbeiter, die einen vermuteten Fall moralischen Fehlverhaltens in ihrem Unternehmen ansprechen (sogenannte ,Whistleblower"), müssen regelmäßig mit negativen Konsequenzen rechnen; Kollegen betrachten sie als "Nestbeschmutzer" und ihre Chancen auf dem Arbeitsmarkt können sinken (vgl. Qusqas/Kleiner 2001). Dem ordnungsethischen Ansatz zu Folge müssen solche Barrieren - etwa durch firmeninterne oder staatliche Regel- und Sanktionssysteme - abgebaut und durch positive Anreize ergänzt werden, um Whistleblowing im wünschenswerten Umfang erwartbar und für die einzelnen moralisch zumutbar zu machen.

In konkreten wirtschafts- und unternehmensethischen Anwendungsfällen ist die Geschichte mit der Etablierung objektiver Regel- und Anreizsysteme aber noch nicht zu Ende. Wer einen vermuteten Korruptionsfall unter Kollegen ungeschickt anspricht, verliert immer noch Ansehen und ist in einer peinlichen Situation, wenn der Verdacht unbegründet war - auch wenn das Ansprechen eines Verdachts prinzipiell richtig und nach Unternehmensrichtlinien erwünscht ist. ${ }^{1}$ Den Verdacht gekonnt anzusprechen, erfordert Taktgefühl, Menschenkenntnis, Durchhaltevermögen und Mut. Wer sich diese Kompetenzen nicht zutraut, wird sich eher passiv verhalten und die Rolle eines Außenstehenden oder Mitläufers weiterspielen. Empirische Studien wie die Untersuchung von MacNab und Worthley (2007) belegen, dass die Bereitschaft zu Whistleblowing mit der subjektiven Einschätzung der eigenen Handlungskompetenz, der Selbstwirksamkeitserwartung zusammenhängt.

Anreizsysteme sind also auf komplementäre Selbstwirksamkeitserwartungen der Akteure angewiesen. Moralische Bildung, Vorbilder und Rollenmodelle stärken diese und sind somit wichtige Elemente bei der Umsetzung von moralischen Normen. Ich werde argumentieren, dass das eine (vielleicht unerwartete) Konsequenz des ordnungsethischen Ansatzes ist, die sich abzeichnet, wenn man ihn psychologisch informiert auf konkrete Fälle anwendet.

\section{Ordnungsethik und die Gestaltung von Anreizstrukturen}

\subsection{Der ordnungsethische Ansatz}

Als Ordnungsethik bezeichne ich die Theorietradition der Wirtschaftsethik, die Karl Homann und sein Schüler in verschiedenen Varianten entwickelt haben (z.B. vgl. Homann/Lütge 2004; Lütge 2007). Nach Pies geht es der Ordnungsethik im Kern um die Umsetzung von anderweitig begründeten Normen unter Wettbewerbsbedingungen: „Im Hinblick auf die typischen Probleme, mit denen sich der Ansatz beschäftigt, ist die Begründung moralischer Anliegen trivial einfach, ihre Implementierung hingegen nicht" (Pies 2010: 251). ${ }^{2}$ Zumindest in liberalen westlichen Gesellschaften ist es

1 Gerade in großen Unternehmen gibt es deswegen Mechanismen, die die Anonymität von Whistleblowern wahren.

2 Dies ist die enge - und für viele Fragen der Wirtschaftsethik angemessene - Charakterisierung des Forschungsprogramms. Das weitere Programm der Ordnungsethik ,als philosophischer Entwurf" (Lütge 2012) zielt auch auf eine Begründung moralischer Normen, typischer Weise durch eine interessenbasierte kontraktualistische Theorie (vgl. Lütge 2013; von Grundherr 2007). 
tatsächlich unstrittig, dass man Korruption, Diskriminierung am Arbeitsplatz oder Kinderarbeit verhindern sollte. Doch dieser Konsens reicht meist nicht, um die Probleme in der Praxis nachhaltig zu lösen.

Diese Lücke zwischen Wertvorstellungen von Individuen und der gesellschaftlichen Umsetzung der entsprechenden Werte ist nach der Analyse der Ordnungsethik systematisch zu erwarten. Unter Wettbewerbsbedingungen seien nämlich vorrangig Interaktionsstrukturen und nicht Einstellungen von Individuen die Ursache für moralisch unerwünschtes Verhalten. Effektive Steuerung von moralischem Handeln müsse daher über eine systematische Änderung der Handlungsbedingungen (sozialer Ordnungen) wirken (vgl. Lütge 2007: 44). Diese Position dient als Ausgangspunkt für meine Argumentation und kann hier nicht weiter kritisch diskutiert werden. ${ }^{3}$

Mir geht es im Folgenden darum, welche Rolle bestimmte Befunde der Psychologie und der experimentellen Ökonomik für die Ordnungsethik spielen. Dafür ist es wesentlich, zwei Aufgaben bei der Gestaltung von sozialen Ordnungen klar zu unterscheiden:

- Die soziale Ordnung muss Anreize bereitstellen, die moralisches Verhalten für den Homo oeconomicus rational machen.

- Die soziale Ordnung muss diese Anreize so zur Verfügung stellen, dass sie für reale Menschen (den Homo psychologicus) effektiv handlungsleitend werden.

In den nächsten beiden Abschnitten begründe ich diese Kriterien und erläutere, warum sie nicht verwechselt werden dürfen.

\subsection{Interaktionslogik in Dilemmastrukturen}

Marktwirtschaftlicher Wettbewerb mobilisiert das Eigeninteresse aller Akteure und macht es als Treiber für gesellschaftlichen Wohlstand verfügbar. Aber nur, wenn das Eigeninteresse an bestimmten Punkten beschränkt wird, ist dieser Wohlstand tatsächlich erreichbar. Moralische Forderungen, etwa das Gebot der Vertragstreue oder das Verbot von Korruption, verlangen eine solche individuelle Beschränkung zum allgemeinen Vorteil. Richtet man diese Forderungen aber direkt an Individuen, verpuffen sie. Denn auch wenn einzelne Akteure zunächst motiviert sind, sich entsprechend einzuschränken, stehen sie letztlich paradoxen Forderungen gegenüber. Wer durch die erwünschte Einhaltung einer Regel in Vorleistung geht, etwa als einziger keine Bestechungsgelder zahlt, verliert im Wettbewerb und handelt gegen das ebenfalls erwünschte Motiv des Eigeninteresses. Auf Dauer erodiert so unter Wettbewerbsbedingungen individuelle moralische Motivation und ist nicht mehr systematisch wiederherzustellen (vgl. ebd.: 250).

Die Ordnungsethik löst dieses Problem, indem sie die moralische Steuerung von Handlungen nicht individuellen Akteuren, sondern der sozialen Ordnung aufträgt. Formale und informelle Institutionen sorgen dann dafür, dass moralisch gewünschtes Handeln mit dem Eigeninteresse der Akteure kompatibel ist. Das Konstrukt des Homo

3 Für die Diskussion über die Angemessenheit der Ordnungsethik sei auf den kürzlich in dieser Zeitschrift erschienenen kritischen Artikel von Aßländer und Nutzinger (2010) sowie die Replik von Pies (2010) verwiesen. 
oeconomicus dient dabei als Prüfstein, um die Anreizkompatibilität von moralischen Forderungen zu testen: Wenn ein institutionelles Arrangement nicht sicherstellt, dass eigeninteressierte, rationale und gut informierte Akteure einer moralischen Regel folgen, dann ist es auf Dauer auch nicht erwartbar, dass reale Akteure des Wirtschaftslebens diese Regel einhalten.

Dieses Argument ist nicht nur ein pragmatischer Hinweis für institutionelles Design. Es zeigt auch, dass moralisch richtiges Verhalten nur dann moralisch gerechtfertigt erwartbar ist, wenn es durch formale und informelle Institutionen abgesichert ist, die sich am idealisierten Messmodell des Homo oeconomicus bewähren. Denn wenn ein Akteur nicht zuverlässig erwarten kann, dass auch seine Interaktionspartner kooperieren, ist es im Kontext des Wettbewerbs nicht zumutbar, dass er selbst kooperiert und sich damit ausbeuten lässt.

Für die bisherige Argumentation sind Befunde der Psychologie und der experimentellen Ökonomik weitgehend irrelevant, da der Homo oeconomicus ein normativ gerechtfertigtes Analyseinstrument ist und keine Beschreibung realer Personen. Würde man ohne diese Idealisierung auf die Welt schauen, wäre die systematische normative Einsicht verdeckt. Wenn die empirische Ökonomik feststellt, dass Menschen de facto kooperieren, kann das nämlich auch ein Indikator dafür sein, dass die Gefahr der Ausbeutung bereits durch informelle Institutionen überwunden ist (vgl. Homann/Lütge 2004: 46). Daraus folgt aber nicht, dass moralisches Verhalten ohne diese Institutionen zumutbar wäre.

\subsection{Handlungsbedingungen für den Homo psychologicus}

Angenommen, ein institutionelles Arrangement bewährt sich am Prüfstein des Homo oeconomicus. Dann ist immer noch offen, ob es auch regelkonformes Verhalten realer Menschen, wie sie die Psychologie beschreibt, sicherstellt. Anders als der Homo oeconomicus wissen psychologische Akteure oft nicht, welche konkreten Verhaltensstrategien sie erfolgreich umsetzen können und welche Wirkungen und Nebenwirkungen bestimmte Handlungen in einem institutionell gestalteten Kontext haben. Sie haben auch regelmäßig falsche Überzeugungen über ihre Einflussmöglichkeiten. Für die konkrete Ausgestaltung von Handlungsbedingungen sind diese Beschränkungen des Homo psychologicus äußerst relevant. ${ }^{4}$

Im Folgenden geht es darum, dass sich die empirisch scharf gezeichneten Menschen nicht unbedingt in ihrer Motivation, wohl aber in ihrem moralischen Kompetenzprofil vom Homo oeconomicus unterscheiden. Diese beiden Charakteristika sollte man systematisch trennen: Wer kompetent, aber nicht motiviert ist, könnte zwar richtig handeln, versucht es aber nicht. Wer motiviert, aber nicht kompetent ist, wird beim Versuch, das Richtige zu tun, erfolglos bleiben. Anzumerken ist, dass ein Mangel an moralischer Kompetenz mittelfristig wie ein Motivationsmangel erscheinen kann,

4 Die Annahme des Homo oeconomicus und des Homo psychologicus sind jeweils einer anderen Fragestellung geschuldet und sind somit komplementäre, nicht widersprüchliche analytische Konstrukte (zur Bedeutung der Fragestellung vgl. Lütge 2007: 49-53). Für sehr hilfreiche Hinweise zur Klärung dieser Abgrenzung und für die Idee, den Homo oeconomicus als Messmodell zu verstehen, danke ich einem anonymen Gutachter. 
denn nach der Erfahrung des Scheiterns wird ein inkompetenter Akteur bald keinen Versuch mehr starten.

Der Homo oeconomicus-Test für Institutionen stellt sicher, dass das Befolgen moralischer Regeln nicht systematisch zu Nachteilen führt und die moralische Motivation psychologischer Akteure nicht systematisch erodiert. Offen ist, ob die Akteure das auch zuverlässig erkennen können und ob sie sich zutrauen, die Regeln korrekt zu befolgen und damit von dem institutionellen Schutz zu profitieren. Wie eingangs erwähnt erfordert Whistleblowing, etwa das Aufdecken eines Korruptionsfalles unter Kollegen, Mut und Durchhaltevermögen sowie die Fähigkeit, mit Frustrationen und Anfeindungen umzugehen - auch wenn bekannt ist, dass es langfristig im Unternehmen honoriert wird. Wenn ein Mitarbeiter in einer solchen Situation nicht in seine Fähigkeit zur Selbstregulierung (ökonomisch gesprochen: in die Fähigkeit zu investieren) vertraut, ist eine Erhöhung der Anreize keine hilfreiche Lösung. Die Stärkung von Selbstvertrauen und das Vermitteln von Selbstmanagementfähigkeiten können viel effizienter sein.

Neben allgemeinen exekutiven Funktionen wie der Selbstregulierungsfähigkeit, sind oft spezifische soziale Handlungskompetenzen entscheidend. Teilnehmer von Zivilcourage-Trainings lernen zum Beispiel ganz konkret, wie sie sich selbst und das Opfer eines Angriffs schützen, indem sie die direkte Konfrontation mit dem Täter meiden. Jonas und Brandstätter betonen „die Bedeutung der Selbstsicherheit und der prompten Verfügbarkeit adäquater Verhaltensweisen zur Ausübung von Zivilcourage - vor allem in Situationen, die mit Angst besetzt sind" (Jonas/Brandstätter 2004: 192). Ziel von Trainings sei es daher nicht, die Angst durch stärkere Motive zu übersteuern, sondern einzuüben, wie man trotz Angst erfolgreich und sicher das Richtige tun könne (vgl. ebd.).

Zusammengefasst: Um moralische Normen umzusetzen, muss man sie durch entsprechend gestaltete Handlungsbedingungen absichern. Das ist die Kernthese der Ordnungsethik. Wenn Institutionen, die solche Bedingungen schaffen, am Prüfstein des Homo oeconomicus scheitern, dann werden sie früher oder später auch am Homo psychologicus scheitern, ohne dass man diesem einen moralischen Vorwurf machen kann. Aber ein Bestehen am Messmodell des Homo oeconomicus zieht nicht notwendigerweise ein Bestehen am Homo psychologicus nach sich. Bei der konkreten Gestaltung von Institutionen muss man deswegen das Kompetenzprofil psychologischer Akteure beachten.

\section{Kompetenz und Selbstwirksamkeitserwartung}

\subsection{Das Konzept der Selbstwirksamkeitserwartung}

Ob ein Ordnungsrahmen die intendierte steuernde Wirkung entfalten kann, hängt also davon $\mathrm{ab}$, ob er reale Akteure anspricht. An dieser Stelle wird das psychologische Konstrukt der Selbstwirksamkeitserwartung für die Ordnungsethik wichtig.

Damit reale Akteure von prinzipiell bestehenden und bekannten Anreizen tatsächlich motiviert werden, wollen sie Antworten auf Fragen wie: „Liegt es in meiner Macht, die Regel zu befolgen? Wie genau mache ich das? Wie kann ich sichergehen, dass es auch 
honoriert wird? Habe ich die Risiken unter Kontrolle?" Die Antworten, die Akteure darauf geben, hängen natürlich auch von ihren tatsächlichen Kompetenzen und der faktischen Effektivität der Anreizmechanismen ab. Doch direkt motivieren die wahrgenommene Kompetenz und die wahrgenommenen Anreize. Für reale Institutionen gilt somit ,esse est percipi'.

Banduras psychologischer Ansatz zur Beschreibung und Messung von Wirksamkeitserwartungen hilft, diese abstrakte Überlegung zu operationalisieren. Das Konstrukt der Selbstwirksamkeitserwartung erfasst die Einschätzung der eigenen Handlungskompetenz. Bandura definiert: „An efficacy expectation is the conviction that one can successfully execute the behavior required to produce the outcomes" (Bandura 1977: 193) Gegeben andere notwendige Bedingungen wie Fähigkeiten und Anreize, wirke die Selbstwirksamkeitserwartung als moderierender Faktor bei der Handlungswahl:

„Given appropriate skills and adequate incentives, however, efficacy expectations are a major determinant of people's choice of activities, how much effort they will expend, and of how long they will sustain effort in dealing with stressful situations" (Bandura 1977: 194).

Es gibt inzwischen starke empirische Evidenz dafür, dass Selbstwirksamkeitserwartungen tatsächlich handlungswirksam sind. Wer eine hohe Erwartung an die eigene Wirksamkeit hat, geht Probleme oder Herausforderungen eher an, ist ausdauernder bei seinen Aktivitäten und kann Rückschläge besser verkraften. In einer großen und viel beachteten Metastudie haben Stajkovic/Luthans (1998) gezeigt, dass Personen mit einer hohen Selbstwirksamkeitserwartung beruflich leistungsfähiger sind.

Selbstwirksamkeitserwartungen sind zum großen Teil erlernt. Bandura nennt vier wesentliche Mechanismen (vgl. Bandura 1977: 195ff). (a) Eigene Erfolgserlebnisse erhöhen den Glauben daran, dass man auch in Zukunft Erfolg haben wird. (b) Durch stellvertretende Erfahrung (,vicarious experience") kann man an den beobachteten Erfolgen anderer lernen, vor allem wenn man ihnen ähnliche Fähigkeiten wie sich selbst zuschreibt - „Das kann ich auch“. (c) Verbale Ermutigung ist schwächer, wirkt aber, wenn sie nicht unrealistisch ist und somit nicht zu Fehlversuchen motiviert. (d) Emotionale Erregung, vor allem Angstreaktionen, können unabhängig von tatsächlichen Misserfolgen dazu beitragen, dass die Selbstwirksamkeitserwartung nachhaltig sinkt.

\subsection{Moralische Selbstwirksamkeit in der Ordnungsethik}

Wie kann man diesen psychologischen Ansatz auf Fragen der Ordnungsethik anwenden? Sind Selbstwirksamkeitserwartungen auch ein wesentlicher Moderator bei Fällen konkreten moralischen Handelns innerhalb von bestehenden Institutionen? An dieser Stelle kann das Beispiel aus der Einleitung systematisch eingeordnet werden. Dort hatte ich darauf hingewiesen, dass ein firmeninternes Regel- und Sanktionssystem für Whistleblowing noch nicht per se garantiert, dass Mitarbeiter wie gewünscht handeln. Denn wer sich nicht zutraut, einen vermuteten Korruptionsfall geschickt anzusprechen, fürchtet trotz dieses Systems Nachteile, etwa einen Ansehensverlust oder eine Schwächung des eigenen firmeninternen Netzwerks. Ungeschickt kann es zum Beispiel sein, das falsche Medium zu wählen, zu viele oder zu wenige Kollegen einzube- 
ziehen, den Missstand nicht überzeugend darzustellen oder den Verdacht aufkommen zu lassen, dass man Kollegen im eigenen Karriereinteresse bloßstellt. So führt eine geringe Erwartung an die eigene Wirksamkeit auch in einem generell bestehenden institutionellen Rahmen dazu, dass man sich zurückhält und nicht eingreift.

Bandura (1977) stellt eine weitere hilfreiche Unterscheidung zur Verfügung: Er grenzt Selbstwirksamkeitserwartungen von Ergebnis-Erwartungen („outcome efficacy") ab. Letztere beziehen sich darauf, dass ein bestimmtes Verhalten ein bestimmtes Ergebnis erzeugt, während sich die Selbstwirksamkeitserwartung darauf bezieht, dass man dieses Verhalten zuverlässig produzieren kann. Institutionen, Regel- und Sanktionssysteme begründen zunächst nur allgemeine Ergebnis-Erwartungen der Art: ,Wenn man Beispiele von Fehlverhalten unter Kollegen auf die richtige Weise anspricht, dann kann man dafür langfristig mit Anerkennung und beruflichem Erfolg rechnen.' Erst die subjektive Selbstwirksamkeitserwartung wandelt aber solche objektive ErgebnisErwartungen in subjektive Anreize um.

In der wirtschaftsethischen Literatur findet sich zuletzt mehrfach der Begriff der ethischen (vgl. Youssef/Luthans 2005: 4) oder auch moralischen Wirksamkeitserwartung, die sich speziell darauf bezieht, mit ethischen Problemen am Arbeitsplatz erfolgreich umzugehen (vgl. May et al. 2013). Im Kontext der Ordnungsethik kann man das noch präziser fassen: Wer eine hohe moralische Selbstwirksamkeitserwartung hat, traut sich $\mathrm{zu}$, moralisch erwünschte und prinzipiell anreizkompatible Verhaltensweisen zu identifizieren und im konkreten Einzelfall so umzusetzen, dass er oder sie tatsächlich von den positiven Folgen profitiert oder zumindest negative Folgen vermeiden kann. Aus der Zivilcourage-Forschung kann man die Einsicht übernehmen, dass dafür fast immer eine sehr konkrete Vorstellung davon notwendig ist, welches Handlungsschema erfolgreich ist und belohnt wird.

\subsection{Empirische Evidenz in der neuesten Forschung}

Das klassische Konzept der Selbstwirksamkeitserwartung hat sich über Jahrzehnte hinweg als gutes Instrument empirischer Forschung bewährt. Psychologische Evidenz aus verschiedenen Forschungsprogrammen bestätigt inzwischen auch, dass Selbstwirksamkeitserwartungen zur Erklärung spezifisch moralischen Verhaltens einen Beitrag leisten können. Der aktuelle Forschungsstand zeigt in dieser Frage einen klaren Trend, auch wenn weitere Studien wünschenswert (und vielversprechend) sind.

Die Bereitschaft zu Whistleblowing steigt mit dem Grad der Selbstwirksamkeitsernartung: MacNab und Worthley (2007) zeigen, dass eine signifikante Korrelation zwischen Selbstwirksamkeit und selbstberichteter Bereitschaft zu Whistleblowing besteht. Untersucht wurden kanadische und US-amerikanische Berufstätige. Selbstwirksamkeitserwartungen und die Bereitschaft zu Whistleblowing wurden mit einem Fragebogen gemessen.

Neben dem zentralen Zusammenhang zwischen Selbstwirksamkeitserwartung und Whistleblowing berichten die Autoren von einem direkten Zusammenhang zwischen Führungserfahrung und Selbstwirksamkeit. Dies kann als Indikator dafür gesehen werden, dass Selbstwirksamkeit auch in diesem Bereich durch Erfolgserlebnisse er- 
lernt wird: Führungskräfte haben die Erfahrung gemacht, dass sie im Arbeitsleben erfolgreich agieren und steuern können.

Ein großer Vorteil dieser Studie ist es, dass sie einen realistischen wirtschaftsethischen Anwendungsfall untersucht und eine große Stichprobe von Personen aus dem aktiven Berufsleben rekrutieren konnte. Weitere Studien zu diesem Thema sind vor allem aus zwei Gründen wünschenswert: (a) Der Effekt ist signifikant, aber nicht stark. ${ }^{5}$ Um seine praktische (und damit wirtschaftsethische) Bedeutung einzuschätzen, ist eine Replikation wünschenswert, die neben einer Absicherung des Ergebnisses klärt, wie sich die Größenordnung des Einflusses zu anderen Einflussgrößen verhält. (b) Ein direktes Verhaltensmaß wurde nicht erhoben. Obwohl berichtete Handlungsabsichten ein guter Prädiktor für tatsächliches Handeln sein können, wäre eine Verhaltensstudie, zum Beispiel im experimental-ökonomischen Labor, wünschenswert.

Mehrere breit gestützte Befunde aus anderen Bereichen der Psychologie lassen aber vermuten, dass sich der Effekt in realem Handeln nachweisen lässt:

Qualitative Studien $₹ u$,moral exemplars": In qualitativen Interviews mit Personen, die im dritten Reich Verfolgte versteckten und retteten, findet Monroe (2011) bei den Rettern systematisch die Überzeugung, dass Hilfsbedürftigkeit einfach zu sehen und Hilfe meist auch einfach zu leisten war. Mitläufer und Außenstehende streiten das hingegen ab: „The basic bystander self-image was one of people who saw themselves as weak, low on efficacy, with little control over their situation" (Monroe 2011: 192). Eine interviewte Außenstehende sagt über sich selbst: „I think to do the right things, and then always do them [sic] the worst things" (ebd.: 193). ${ }^{6}$

Für die Ordnungsethik sind diese historischen Fälle hoch interessant. Sie spielten sich im Rahmen der starken externen Anreizstruktur ab, die das repressive nationalsozialistische System aufgebaut hatte. Individuen nahmen diesen institutioneller Rahmen aber sehr unterschiedlich wahr. Die individuelle Einschätzung moderierte damit die tatsächliche Wirksamkeit der Handlungsbedingungen für die einzelnen Personen.

Monroes qualitative Methode ermöglicht ein reiches Verständnis der Mechanismen, die tatsächlichem moralischem Handeln zugrunde liegen. Für eine qualitative Studie ist Monroes langjähriges Forschungsprogramm mit hunderten von Interviews sehr breit belegt und läuft nicht Gefahr, Ausnahmen zu verallgemeinern. Obwohl sie Banduras Konzept nicht explizit verwendet und der Methode entsprechend kein Standardmaß für Selbstwirksamkeit einsetzt, passen ihre Beobachtungen sehr gut zu Banduras Theorie.

5 Die Autoren berichten von einem signifikanten Pfadkoeffizienten von 17 zwischen den beiden latenten Variablen ,self-efficacy" und ,internal whistleblowing" in einem gut passenden Strukturgleichungsmodell (vgl. MacNab/Worthley 2007: 414).

$6 \quad$ Monroe verwendet an mehreren Stellen auch das Konzept der Kontrollüberzeugung (locus of control'), um Helfer von Unterstützern und Außenstehenden zu unterscheiden: „Rescuers exhibited an internal locus of control over their fate; bystanders and supportes of genocide tended more toward an external locus" (Monroe 2011: 203). Obwohl es Unterschiede zwischen den theoretischen Konzepten der Kontrollüberzeugung und der Selbstwirksamkeitserwartung gibt, sind die Konstrukte empirisch kaum zu trennen, wie Judge et al. (2002) zeigen. 
Selbstwirksamkeit und Mobbing-Rollen: Mehrere aktuelle Studien zu Determinanten von Verhalten in Mobbing-Situationen in Schulen zeigen, dass sich Selbstwirksamkeitserwartungen signifikant zwischen Verteidigern und Außenstehenden unterscheiden. Verteidiger greifen aktiv in das Geschehen ein und helfen dem gemobbten Opfer, während Außenstehende sich zwar nicht an den aggressiven Handlungen beteiligen, aber auch nicht helfen. In einer aktuellen einschlägigen Studie berichten Thornberg und Jungert (2013), dass Selbstwirksamkeit deutlich positiv mit Verteidigerverhalten und negativ mit Außenstehenden-Verhalten korreliert ist. ${ }^{7}$ Allerdings erhoben sie Selbstwirksamkeit mit nur zwei Fragebogenitems. Sie bestätigen das Ergebnis von Gini et al. (2008), die mit einer längeren Skala und durch ,peer-nomination' genauer erhobenen Mobbing-Rollen eine um Geschlechtseffekte bereinigte positive Korrelation von Selbstwirksamkeit und Verteidigerverhalten finden, während Verhalten von Außenstehenden negativ damit korreliert ist. ${ }^{8}$ Weitere stützende Befunde finden sich bei Pöyhönen et al. (2010) sowie bei Pozzoli und Gini (2013).

Während Monroe Ausnahmesituationen untersucht, beschäftigen sich die MobbingStudien mit viel alltäglicheren Kontexten. Beide Forschungsprogramme kommen aber prinzipiell zu ähnlichen Ergebnissen. Mobbing in Schulen tritt in ähnlicher Weise sehr oft auf und eignet sich daher gut, um reproduzierbare und valide Daten über die Determinanten moralischen Verhaltens zu erheben.

Für den wirtschaftsethischen Kontext sind die Studien direkt und indirekt einschlägig. Mobbing am Arbeitsplatz ist trotz aller Unterschiede prinzipiell vergleichbar mit Mobbing in der Schule (vgl. Smith et al. 2003). Aber die Ergebnisse sind auch für eine breitere Spanne von wirtschaftsethischen Problemen einschlägig. Für viele wirtschaftsethische Fragen ist genau die Unterscheidung zwischen Außenstehenden und denjenigen, die eingreifen, relevant. Diskriminierung am Arbeitsplatz, informell institutionalisierte Prozesse für Korruption oder systematisch akzeptierte ,Kavaliersdelikte' wie falsche Spesenabrechnungen sind nur möglich, wenn viele wegschauen und eben nicht eingriffen wird.

Mit dem nun entwickelten Instrumentarium ist es möglich, die ordnungsethische Theorie an zwei (zusammenhängenden) Punkten zu differenzieren, die bei Kritikern häufig Unbehagen hervorrufen. In beiden Punkten muss die Ordnungsethik keine radikale Frontstellung einnehmen, sondern kann intuitiv plausible Thesen ihrer Kritiker zumindest teilweise theoretisch integrieren, indem sie sie nicht auf die Motivation, sondern auf die Kompetenzen und Wirksamkeitserwartungen moralischer Akteure bezieht.

7 Die Korrelationen sind signifikant und mit Koeffizienten von .47 bzw. - -.44 und gehören damit eindeutig zu den stärkeren Effekten, von denen in der Mobbing-Forschung berichtet wird.

8 Gini et al. 2008 berichten von signifikanten, aber schwächeren Kotrelationen mit Koeffizienten von .20 bzw. -27 . 


\section{Implikationen für die Theoriebildung der Ordnungsethik}

\subsection{Aufbau von Kompetenz und Wirksamkeitserwartung als Teil der Gestaltung sozialer Ordnungen}

Homann formuliert in einem Vortrag pointiert: „Menschen verhalten sich gemäß ihren Anreizen. Anreize werden nur (sic!) durch andere, stärkere Anreize überwunden, nicht durch Moral“ (Homann 2007: 31). Solche Formulierungen rufen Kritiker auf den Plan: Die Ordnungsethik zeichne den moralischen Akteur als unreflektierten Reiz-Reaktionsapparat, dem allein mit einem komplementären Sanktionsmechanismus beizukommen sei (vgl. Aßländer/Nutzinger 2010: 242). Dass aber moralische Bildung des Individuums keine Rolle bei der Umsetzung moralischer Normen spiele, ist aus einer lebensweltlichen Perspektive heraus nicht plausibel und läuft alltäglichen Erfahrungen zuwider.

Eine psychologisch informierte Ordnungsethik kann diesen Einwand durch die Differenzierung von Motivation, Kompetenz und Wirksamkeitserwartung einordnen und relativieren. Damit ein prinzipiell bestehendes institutionelles Arrangement wirksam wird und Verhalten zum wechselseitigen Vorteil lenkt, müssen nämlich weitere Bedingungen erfüllt sein.

Moralische Akteure brauchen auch bei gegebenen Anreizstrukturen konkrete Handlungskompetenz: Ein Fußballspieler, der Fouls vermeiden möchte, aber die entsprechenden motorischen Fähigkeiten nicht mitbringt, wird trotzdem oft (versehentlich oder aus Verzweiflung) foulen. Ebenso wird er nur dann ein guter Teamspieler sein, der die Mannschaftskollegen mit Pässen versorgt, wenn er genau zuspielen kann. Das ist in diesem Beispiel trivial; bei Fragen moralischen Handelns wird regelmäßig übersehen, dass es in komplexen sozialen Situationen stattfindet, die hohe soziokognitive Kompetenzen und ein Arsenal an souverän beherrschten Handlungsschemata erfordern. Verwiesen sei hier noch einmal darauf, dass Zivilcourage-Trainings diese Kompetenzen schulen.

Moralische Akteure brauchen auch bei gegebenen Anreizstrukturen moralische Urteilskompetenz: Ein Fußballspieler, der ohne die Regeln des Spiels zu kennen versucht, gelbe Karten zu vermeiden, wird sich nicht souverän im Spiel bewegen, viele unnötige Strafen kassieren und schnell aufgeben. Genauso müssen moralische Akteure auch in einem bestehenden institutionellen Rahmensystem mitdenken und ihre moralische Urteilskraft anwenden. Wer nicht moralisch urteilen kann, welche Handlung in einer Situation richtig ist, hat geringe Chancen, die gesetzten Anreize zu erreichen. Für psychologisch realistische Menschen sind die meisten Anreizstrukturen nur als Sanktion für die moralischen Normen zu verstehen. Die Normen liefern das passende kognitive Schema. Moralische Urteilskraft ist somit eine wichtige und erlernbare individuelle Eigenschaft, die Anreize für den Homo psychologicus erreichbar macht. In die Ordnungsethik hat dieser Gedanke in ähnlicher Form bereits mit der Forderung von Lin-Hi und Suchanek Eingang gefunden, das „Spielverständnis“ der einzelnen Akteure als wichtige 
Komponente eines Selbstbindungssystems zu berücksichtigen (vgl. Lin-Hi/Suchanek 2009: 25) ${ }^{9}$

Bei kompetenten moralischen Akteuren ist die Selbstwirksamkeitserwartung das Bindeghied zwischen Anreizen und Handeln: Anreize werden auch bei handlungs- und urteilskompetenten Akteuren nicht automatisch handlungswirksam. Nur wer an die Zuverlässigkeit des eigenen Urteils glaubt und darauf vertraut, dass er auch in der Lage ist, das geforderte gekonnt umzusetzen, kann sicher sein, dass er von den Anreizen auch profitieren wird oder zumindest keine Nachteile zu erwarten hat. Es sei daran erinnert, dass Selbstwirksamkeitserwartungen deutlich zwischen Helfern und Außenstehenden differenzieren, auch wenn beide im selben Kontext (unter den selben objektiven Anreizen) entscheiden. Selbstwirksamkeitserwartungen sind genauso wie Handlungs- und Urteilskompetenz lernbar.

Normimplementiernng, die auf moralische Bildung setrt, hat oft die geringsten gesellschaftlichen Kosten: Das Bild, aus Sicht der Ordnungsethik bestehe die einzige Aufgabe wirtschaftsethischer Praxis in der Etablierung eines formalen Straf- und Belohnungsapparates (z.B. staatliche Regeln mit Polizei und Justiz oder firmeninterne Anreizsysteme), ist ohnehin falsch (vgl. Pies 2010: 252). Die Ordnungsethik sucht nach einer Möglichkeit, Normen mit möglichst geringen Kosten für alle Mitglieder der Gesellschaft umzusetzen. Aus den drei eben erläuterten Punkten folgt: Wenn den realen Akteuren moralische Kompetenzen oder auch nur der Glaube an diese Kompetenzen fehlen, kann das nicht oder nur auf sehr ineffiziente Weise mit roher Gewalt durch stärkere objektive Anreize kompensiert werden. Bildungsmaßnahmen sind oft der ökonomisch effizientere und gesellschaftlich rechtfertigbare Weg.

In konkreten Problemfällen wird die Ordnungsethik aus ihrer eigenen Logik heraus immer vorschlagen zu prüfen, ob nicht im Prinzip bereits hinreichend starke Anreizsysteme bestehen, die nur nicht wirksam werden, weil konkrete Kompetenzen oder Vertrauen auf diese Kompetenzen fehlen. Kompetenzaufbau und Förderung der Selbstwirksamkeitserwartung können günstige Mittel sein, die Effizienz von Institutionen zu steigern. Drei Aufgaben folgen in wirtschaftsethischen Anwendungskontexten für alle, die Handlungsbedingungen für moralisch erwünschtes Verhalten gestalten: Zusätzlich zur Gestaltung von objektiven (am Homo oeconomicus getesteten) Anreizen, sollten sie

- durch gute Kommunikation Ergebnis-Erwartungen herstellen, also Regeln und Anreize klar und an konkreten Beispielen kommunizieren,

- $\quad$ spezifische Handlungs- und Urteilskompetenz aufbauen, etwa durch Trainingsmaßnahmen und das Bereitstellen von angstfreien Lernchancen, und

- Selbstwirksamkeitserwartungen fördern. Empirische Studien haben gezeigt, dass Bildungsmaßnahmen (vgl. May et al. 2013; Nelson et al. 2012) und der Aufbau einer entsprechenden Kultur (vgl. Shacklock et al. 2011) die moralische Selbstwirksamkeit effektiv erhöhen.

9 Auch offene oder unvollständige Verträge, die in der Ordnungsethik vor allem bei unternehmensethischen Fragen eine wichtige Rolle spielen, sind nur möglich, wenn die Vertragspartner in diesem Sinne mitdenken und beurteilen können, wie der Vertrag sinngemäß erfüllt werden sollte (vgl. Lütge 2007: 47-49). 
Diese pädagogische Aktivität ist nicht mit Werteerziehung im Sinne von Aufbau selbstloser Motivation zu verwechseln und kompatibel mit dem ordnungsethischen Programm. Es bleibt ein - nun schärfer umrissener - Konfliktpunkt mit Kritikern bestehen, die auf eine rein motivationale Stärkung von Werten setzen. ${ }^{10}$

\subsection{Die Rolle von Vorbildern in der Ordnungsethik}

Die Ordnungsethik hat es oft als eine (Fehl-)Meinung anderer ethischer Ansätze gesehen, dass sie Vorbildern eine wichtige Rolle zuschreiben. Bei Homann findet sich der Glaube an Vorbilder in einem „Sündenregister“, das er - in rhetorischer Absicht - für ein „Standardmodell der Ethik“" (Homann 2008: 2) aufstellt. Die Vertreter dieses Modells argumentierten ,der schwache Wille“ müsse ,gestärkt werden: durch Erziehung, durch Vorbilder“ (Homann 2008: 3). Damit verfehlten sie die wesentliche Stellschraube bei der Umsetzung moralischer Werte, nämlich die Änderung der Handlungsbedingungen. Auch das widerspricht der lebensweltlichen Praxis, sei es in der Erziehung von Kindern oder bei der Führung von Mitarbeitern.

Aus dem Kontext wird klar, dass Homann die These auf das Problem des Motivationsaufbaus bezieht. Vorbilder, die den Verzicht auf eigene Interessen vorlebten, könnten unter dem moral-erodierenden Wettbewerb langfristig bestenfalls bei Ausnahmepersönlichkeiten eine Wirkung haben. In dieser Lesart schließt die These nicht aus, dass Vorbilder auf eine andere Weise wirken und zur Umsetzung von Moral beitragen können. ${ }^{11}$ Für die Ordnungsethik wichtig sind vor allem zwei Funktionen: Vorbilder können (a) unklare Situationen disambiguieren und sie können (b) Lernprozesse auslösen, die zu einer höheren moralischen Selbstwirksamkeitserwartung führen.

Vorbilder disambiguieren unklare Situationen: Mitarbeiter von Unternehmen bewegen sich in einer Vielzahl sich überlagernder sozialer Regelsysteme. Formale Verhaltenskodizes und Richtlinien sind nur eines dieser Systeme. Informelle Praktiken können Forderungen stellen, die mit ihnen in Widerspruch treten. In einzelnen Bereichen eines Unternehmens kann das Zahlen von Bestechungsgeldern üblich sein und von einzelnen implizit erwartet werden, auch wenn es eine offizielle (sanktionsbewehrte) Unternehmensrichtlinie gibt, die es verbietet. Zudem sind vermutlich (pseudo-)rechtfertigende Narrative im Umlauf: ,Die Wettbewerber machen es auch, und sogar in größerem Maßstab.' ,Wenn wir nicht mitmachen, bekommen wir keine Aufträge. So läuft das Geschäft eben.' Mitarbeiter haben dann zwei ähnlich kohärente Bewertungssysteme zur Auswahl. Ihnen ist zudem unklar, mit welchen Folgen sie zu rechnen haben, wenn sie gegen den direkten Vorgesetzten, aber im Sinne der Richtlinie entscheiden. Können sie darauf vertrauen, dass die Richtlinie beim oberen Management wirklich ernst

10 Kein Konflikt besteht übrigens mit Moralpsychologen wie Lind, der zwar schon im Buchtitel behauptet: „Moral ist lehrbar", dann aber klarmacht, dass er damit auf Anwendungskompetenz, nicht auf Werteerziehung zielt. Letztere habe sich empirisch über Jahrzehnte hinweg als ineffizient erwiesen (vgl, Lind 2003: 34f.).

11 Die klare Formulierung dieses Gedankens und der folgenden Ausführung verdanke ich einer Anregung von Karl Homann. 
genommen wird? Können sie in dem Unternehmen ohne Korruption Karriere machen? Für die Mitarbeiter sind sowohl die Regeln als auch das Anreizsystem unklar.

Das Verhalten eines Vorbilds kann die Deutung einer solchen unklaren Situation erleichtern. Wenn eine Abteilungsleiterin sichtbar vorlebt, dass man ohne das Zahlen von Bestechungsgeldern erfolgreich im Vertrieb der Produkte sein und Karriere machen kann, gibt sie den Mitarbeitern dadurch eine glaubwürdige Information. In Banduras Terminologie stellt ihr Verhalten Ergebnis-Erwartungen her. Die Mitarbeiter wissen: Wenn ich mich so verhalte wie sie, dann kann ich offensichtlich mit einem vorteilhaften Ausgang rechnen. ${ }^{12} \mathrm{Zu}$ vermuten ist auch, dass im Gegenzug passives oder unmoralisches Verhalten von Vorbildern (Führungskräften) die Ambiguität der Situation verstärkt. So mag ein Mitarbeiter denken: ,Wenn nicht einmal die prädestinierten und potenziell besser informierten Vorgesetzten mitmachen, wird es schon einen guten Grund dafür geben.'

Vorbilder stärken die Selbstwirksamkeitsenwartung: Bandura nennt stellvertretende Erfahrung (,vicarious experience") als einen wesentlichen Lernmechanismus für Selbstwirksamkeitserwartungen:

„Seeing others perform threatening activities without adverse consequences can generate expectations in observers that they too will improve if they intensify and persist in their efforts" (Bandura 1977: 197).

Im oben genannten Fall mag ein Mitarbeiter zwar überzeugt sein, dass kompetente Opposition gegen die etablierte Korruptionspraxis für ihn richtig und letztlich vorteilhaft ist, fehlt es ihm aber an Selbstwirksamkeitserwartung, so traut er sich das nicht zu. Er kann daran zweifeln, dass er als Whistleblower die richtigen Ansprechpartner und die richtigen Worte findet. Vielleicht ist er auch nicht sicher, dass er eine Durststrecke durchhalten kann, in der er lernen muss, seine eigenen Kunden ohne die Zahlungen zu halten. Beobachtet er ein Vorbild, das all das erfolgreich tut und das ihm insgesamt relativ ähnlich ist, wird das seine Vorbehalte reduzieren.

$\mathrm{Ob}$ prinzipiell bestehende Anreizstrukturen wirksam werden, hängt also auch davon $\mathrm{ab}$, ob Individuen erfolgreich die zugrundeliegenden Regeln vorleben. Die Vorbilder zeigen aber nicht, wie man eigene Interessen zurückstellt, sondern sie weisen darauf hin, wie man prinzipiell bestehende Anreizstrukturen konkret für sich nutzbar machen kann.

Dieser Zug ermöglicht der Ordnungsethik, die im öffentlichen Diskurs oft betonte Bedeutung von Vorbildern und Rollenmodellen theoretisch einzuordnen. Wirksame Vorbilder motivieren nicht zur Nachahmung selbstloser Handlungen. Vielmehr klären

12 Dass die Eindeutigkeit einer moralisch relevanten Situation einen starken Einfluss auf tatsächlich gezeigtes moralisches Verhalten hat, zeigten bereits Clark und Word (1974) mit einer Studie, in der sie Hilfeverhalten bei einem simulierten Arbeitsunfall untersuchten. Brauchte das Opfer eindeutig Hilfe, halfen alle Versuchspersonen. Diese Quote fiel bereits bei moderater Ambiguität drastisch (bei Personen, die alleine unterwegs waren, auf 36 Prozent). 
sie uneindeutige oder schwierige Situationen und zeigen, dass und wie man mit moralischem Verhalten im jeweiligen Kontext (etwa beruflich) erfolgreich sein kann.

\section{Perspektiven für die weitere Forschung}

Meine Argumentation kann man als Vorschlag verstehen, die allgemeine Ordnungsethik in Anwendungsfällen durch eine psychologisch informierte angewandte Ordnungsethik fortzusetzen. Diese fragt, unter welchen Bedingungen generell wünschenswerte und anreizkompatible Regeln bei psychologisch präzise beschriebenen Menschen, etwa den Mitarbeitern und Mitarbeiterinnen eines Unternehmens, handlungswirksam werden. Dies ergänzt das allgemeine ordnungsethische Programm durch die komplementäre Frage nach einem wirksamen moralischen ChangeManagement. Ich habe empirisch gestützt argumentiert, dass zu einem solchen Veränderungsprogramm sowohl der Aufbau neuer Kompetenzen als auch die Stärkung der subjektiven moralischen Selbstwirksamkeitserwartung gehören. Ich habe abschlieBend gezeigt, dass es eine wichtige Aufgabe von Führungskräften ist, entsprechende Verhaltensmuster vorzuleben, Lernchancen für Mitarbeiter zu schaffen und psychologisch wirksam die Angst vor dem Scheitern bei der Umsetzung von Normen zu nehmen. Gelingt das, können auch relativ schlanke formale Anreizsysteme und Institutionen wie Richtlinien, Prozesse und monetäre Bonussysteme ihre Wirkung effektiv entfalten.

Es gibt bereits gute empirische Belege für die Bedeutung der Selbstwirksamkeitserwartung bei der Umsetzung von moralischen Regeln. In Abschnitt 3.3 habe ich einschlägige Studien und Forschungsprogramme genannt. An vielen Stellen eröffnen sich aber Perspektiven für weitere Forschung. Weitere Studien mit einem spezifisch wirtschaftsethischen Paradigma sind wünschenswert. Zudem ist es wichtig, direkt zu untersuchen, wie Selbstwirksamkeitserwartungen in diesen Kontexten mit tatsächlichem Verhalten zusammenhängen. Denkbar sind hier zum Beispiel Verhaltensexperimente im experimentalökonomischen Labor oder auch Feldstudien. Wenn die bisherigen Ergebnisse validiert und differenziert werden, wird die empirische Forschung einen direkten Einfluss auf die Theoriebildung und Anwendung der Ordnungsethik haben.

\section{Literaturverzeichnis}

Aßländer, M. S./Nutzinger, H. G. (2010): Der systematische Ort der Moral ist die Ethik!, in: Zeitschrift für Wirtschafts- und Untemehmensethik, Jg. 11/Heft 3, 226-248.

Bandura, A. (1977): Self-efficacy: Toward a Unifying Theory of Behavioral Change, in: Psychological Review, Vol. 84/No. 2, 191-215.

Clark, R. D./Word, L. E. (1974): Where is the Apathetic Bystander? Situational Characteristics of the Emergency, in: Journal of Personality and Social Psychology, Vol. 29/No. 3, $279-287$.

Gini, G./Albiero, P./Benelli, B./Altoè, G. (2008): Determinants of Adolescents' Active Defending and Passive Bystanding Behavior in Bullying, in: Journal of Adolescence, Vol. 31/No. 1, 93-105.

von Grundherr, M. (2007): Moral aus Interesse: Metaethik der Vertragstheorie, Berlin, New York: de Gruyter. 
Homann, K. (2007): Moral und ökonomisches Gesetz, in: Streeck, W./Beckert, J. (Hrsg.): Moralische Voraussetzungen und Grenzen wirtschaftlichen Handelns, MPIfG Working Paper, Köln: Max-Planck-Institut für Gesellschaftsforschung, 23-36.

Homann, K. (2008): Was bringt die Wirtschaftsethik für die Ethik: Abschiedsvorlesung an der Ludwig-Maximilians-Universität München am 17. Juli 2008, Diskussionspapier Nr. 2008-4, hrsg. vom Wittenberg-Zentrum für Globale Ethik, Wittenberg.

Homann, K./Lütge, C. (2004): Einführung in die Wirtschaftsethik, Berlin, Münster: Lit.

Jonas, K. J./Brandstätter, V. (2004): Zivilcourage, in: Zeitschrift für Sozialpsychologie, Jg. 35/Heft 4, 185-200.

Judge, T. A./Ereq, A./Bono, J. E./Thoresen, C. J. (2002): Are Measures of Self-esteem, Neuroticism, Locus of Control, and Generalized Self-efficacy Indicators of a Common Core Construct?, in: Journal of Personality and Social Psychology, Vol. 83/No. 3, 693-710.

Iind, G. (2003): Moral ist lehrbar: Handbuch zur Theorie und Praxis moralischer und demokratischer Bildung, München: Oldenbourg.

Lin-Hi, N./Suchanek, A. (2009): Eine wirtschaftsethische Kommentierung der Finanzkrise, in: Forum Wirtschaftsethik, Jg. 17/ Heft 1, 20-27.

Lïtge, C. (2007): Was hält eine Gesellschaft zusammen? Ethik im Zeitalter der Globalisierung, Tübingen: Mohr Siebeck.

Lütge, C. (2012): Ordnungsethik als philosophischer Entwurf, in: Lütge, C (Hrsg.): Wirtschaftsethik ohne Illusionen: Ordnungstheoretische Reflexionen, Tübingen: Mohr Siebeck, 89-110.

Lütge, C. (2013): The Idea of a Contractarian Business Ethics, in: Lütge, C. (Ed.): Handbook of the Philosophical Foundations of Business Ethics, Dordrecht: Springer Netherlands, 647-658.

MacNab, B. R/Wortbley, R (2007): Self-Efficacy as an Intrapersonal Predictor for Internal Whistleblowing: A US and Canada Examination, in: Journal of Business Ethics, Vol. 79/No. 4, 407-421.

May, D. R.Luth, M. T./Schwoerer, C. E. (2013): The Influence of Business Ethics Education on Moral Efficacy, Moral Meaningfulness, and Moral Courage: A Quasi-experimental Study, in: Journal of Business Ethics, vorab online erschienen, heruntergeladen am 6.11.2013 von http://link.springer.com/article/10.1007\%2Fs10551-013-1860-6.

Monroe, K. R. (2011): Ethics in an Age of Terror and Genocide: Identity and Moral Choice, Princeton, N.J.: Princeton University Press.

Nelson, J. K./Poms, L. W./Wolf, P. P. (2012): Developing Efficacy Beliefs for Ethics and Diversity Management, in: Academy of Management Learning \& Education, Vol. 11/No. $1,49-68$.

Pies, I. (2010): Karl Homanns Programm einer ökonomischen Ethik, in: Zeitschrift für Wirtschafts-und Unternehmensethik, Jg. 11/Heft 3, 249-261.

Pöybönen, V./Juvonen, J./Salmivalli, C. (2010): What Does it Take to Stand Up for the Victim of Bullying?: The Interplay Between Personal and Social Factors, in: Merrill-Palmer Quarterly, Vol. 56/No. 2, 143-163.

Pozzoli, T./Gini, G. (2013): Why Do Bystanders of Bullying Help or Not? A Multidimensional Model, in: The Journal of Early Adolescence, Vol. 33/No. 3, 315-340.

Qusqas, F./Kleiner, B. H. (2001): The Difficulties of Whistleblowers Finding Employment, in: Management Research News, Vol. 24/No. 3-4, 97-100. 
Shacklock, A. H./Manning, M./Hort, L. (2011): Ethical Climate Type, Self-efficacy, and Capacity to Deliver Ethical Outcomes in Public Sector Human Resource Management, in: Journal of New Business Ideas \& 'Trends, Vol. 9/No. 2, 34-39.

Smith, P. K./Singer, M./Hoel, H./Cooper, C. L. (2003): Victimization in the School and the Workplace: Are there any Links?, in: British Journal of Psychology, Vol. 94/No. 2, 175-188.

Stajkovic, A. D./Luthans, F. (1998): Self-efficacy and Work-related Performance: A Metaanalysis, in: Psychological Bulletin, Vol. 124/No. 2, 240-261.

Thornberg, R./Jungert, T. (2013): Bystander Behavior in Bullying Situations: Basic Moral Sensitivity, Moral Disengagement and Defender Self-Efficacy, in: Journal of Adolescence, Vol. 36/No. 3, 475-483.

Youssef, C. M./Luthans, F. (2005): A Positive Organizational Behavior Approach to Ethical Performance, in: Giacalone, R. A./Jurkiewicz, C. L./Dunn, C. (Eds.): Positive Psychology in Business Ethics and Corporate Responsibility, Charlotte: Information Age Publishing, 1-22. 